\title{
Nonlinear Optical Properties, Optical Limiting and Optical Switching of Ag Nanoparticles Prepared by a Green Synthetic Method
}

\author{
Fryad Z. Henari ${ }^{1}$, Hacene Manaa ${ }^{2}$ \\ ${ }^{1}$ Department of Medical Sciences, Royal College of Surgeons in Ireland, Medical University of Bahrain, Busaiteen, Kingdom of \\ Bahrain \\ ${ }^{2}$ Physics Department, College of Science, University of Bahrain, Skhir, Kingdom of Bahrain \\ Email: hmanaa @gmail.com
}

How to cite this paper: Henari, F.Z. and Manaa, H. (2018) Nonlinear Optical Properties, Optical Limiting and Optical Switching of Ag Nanoparticles Prepared by a Green Synthetic Method. Optics and Photonics Journal, 8, 235-246.

https://doi.org/10.4236/opj.2018.87020

Received: June 6, 2018

Accepted: July 14, 2018

Published: July 17, 2018

Copyright $\odot 2018$ by authors and Scientific Research Publishing Inc. This work is licensed under the Creative Commons Attribution International License (CC BY 4.0).

http://creativecommons.org/licenses/by/4.0/

\begin{abstract}
Silver nanoparticles were synthesized using an aqueous extract of cinnamon berks (cinnamon cassia) as a reducer and stabilizer. The synthesized silver nanoparticles were characterized using UV-Vis spectrometry and Transmission Electron Microscopy (TEM), which revealed the nano nature of the particles. Nonlinear absorption and nonlinear refraction were measured using a Z-scan technique at different wavelengths with $\mathrm{CW}$ lasers. The third order nonlinear susceptibility was found to be between (1.5 to 22$) \times 10^{-14} \mathrm{~m}^{2} / \mathrm{V}^{2}$. The nonlinear property of the synthesized silver nanoparticles was used to demonstrate optical limiting and all-optical switching. An optical limiting threshold was found to be $0.1 \mathrm{~mW}$ at $632.8 \mathrm{~nm}$.
\end{abstract}

\section{Keywords}

Green Synthesis, Ag Nanoparticles, Nonlinear Optical Properties, Optical Limiting, Optical Switching

\section{Introduction}

Metal nanoparticles such as silver and gold have attracted a significant interest relative to counterparts due to their wide range of applications in many fields, such as optoelectronic, medical diagnostic imaging and therapy, anti-bacterial and as a catalysis. Different methods have been reported for synthesizing silver nanoparticles (AgNPs), either physical or chemical methods [1] [2] [3]. Chemical reduction is the most common method which involves the reduction of the silver precursor in the liquid phase using sodium citrate. However, their disposal 
raises environmental issues due to cytotoxicity provoked by citrate. Hence, it is essential to develop environmentally friendly techniques for synthesis of nanoparticles. Nanoparticles made by employing green-chemistry based on the extract of compounds from plants and microbes potentially eliminate the environmental issues. These techniques are simple and less expensive and nanoparticles of different sizes and shapes can be produced in large scale. Green synthesis based on plants and plant extracts such as Hibiscus Cannabinus leaf [4], Curcumin [5], and Henna [6] have been successfully used for synthesizing metal nanoparticles. Extracts from plants usually contain sugars, terpenoids, polyphenols, alkaloids, phenolic acids, and proteins, are excellent reducing agents used in the synthesis of nanoparticles.

Metal nanoparticles such as Ag and Au synthesized by chemical reduction have been investigated for their large third order, nonlinear optical properties and their considerable fast time response, which are important for applications in optical devices such optical limiting and optical switching. The enhanced nonlinear optical properties of such materials are due to the amplification of local electric fields at the surface of the metal particles at the range of surface Plasmon resonance frequency. The nonlinear optical properties are strongly dependent on the size, shape and dielectric properties of metals and its surroundings [7].

This study reports on the synthetization of Ag NPS using cinnamon berks. Two different methods were used: the normal heating method and the normal microwave heating. The nanoparticles were confirmed by UV-Vis spectrometry and Transmission Electron microscopy (TEM). The nonlinear absorption and nonlinear refractive index of silver nanoparticles were investigated at different CW laser wavelengths. The nonlinear optical properties were utilized to demonstrate optical limiting and optical switching.

\section{Materials Synthesizes}

Silver Nitrate was bought from Sigma Aldrich chemicals, Germany. Cinnamon berks were bought from the local shop. The cinnamon berks were grained with a blender and sieved. A $2.5 \mathrm{~g}$ of grained cinnamon was dissolved in $100 \mathrm{ml}$ of double-distilled water. Then, this solution was boiled for 5 minutes and filtered twice with the help of what man No. 1 filter paper.

Ag nano particles were synthesized according to the procedure given in [8]. Briefly, a $42.1 \mathrm{mg}$ of $\mathrm{AgNO}_{3}$ was dissolved in $100 \mathrm{ml}$ of distilled water to prepare a $1 \mathrm{mM} \mathrm{AgNO}$ solution. $1 \mathrm{ml}$ of cinnamon was mixed into $50 \mathrm{ml}$ of the $\mathrm{AgNO}_{3}$ solution. The mixture was divided into two parts. One part of the solution was placed in a microwave oven for 20 seconds and the other part was stirred and boiled for 2 minutes. In both cases, the reduction took place as indicated by a yellowish colour of the solution. The solution was found to be stable for a month with no colour changes and showed little or no precipitation.

\section{Characterization}

Initial characterization of the silver nanoparticles was carried out using a 
UV-Vis spectrometer (Shimadzu UV-1800). It is well known that the metal nanoparticles exhibit distinctive optical properties due to the combined oscillations of conduction band electrons in resonance with the incident wavelength, which is known as the Surface Plasmon Resonance (SPR) band. The identity of the silver nanoparticles was confirmed by recording the absorption spectra over 200 $600 \mathrm{~nm}$ after 30 minutes of the heating process. Figure 1 shows UV-Vis absorption spectra of silver nanoparticles for boiling and Microwave Heating. The observed SPR peaks around $433 \mathrm{~nm}$ is an indication of the formation of silver nanoparticles. The full wave at half maximum (FWHM) of Plasmon peak gives information regarding the particles' size. It can be seen from Figure 1 that the FWHM of SPR for nanoparticles synthesized by the boiling method is larger than the one of nanoparticles synthesized by Microwave method. This indicates that the size of the nanoparticles is larger for the boiling case than the MW case, which was confirmed by TEM images and size distribution of nanoparticles analyzed using ImageJ 1.5J software see Figure 2. The sharp peaks and symmetrical nature of the absorption spectrum indicate the formation of spherical nanoparticles, which was confirmed by TEM images. The stability of the samples was monitored over one month by recording the absorption spectra. During this period, the absorption band was constantly observed around $433 \mathrm{~nm}$, confirming the preservation of nanoparticles in the solution. The broadening of the SPR band and a small shift in absorption peak of SPR (redshift) were observed over the time Figure 3. The shift in the absorption peak is an indication of the increase in sizes of nanoparticles [9]. It was observed that the SPR peak at $433 \mathrm{~nm}$ increased as a function of time up to 14 days. After that time, a very small variation in the SPR peak was observed. This indicates the stability of concentration and sizes of nanoparticles [4]. This stability may arise from a balance between

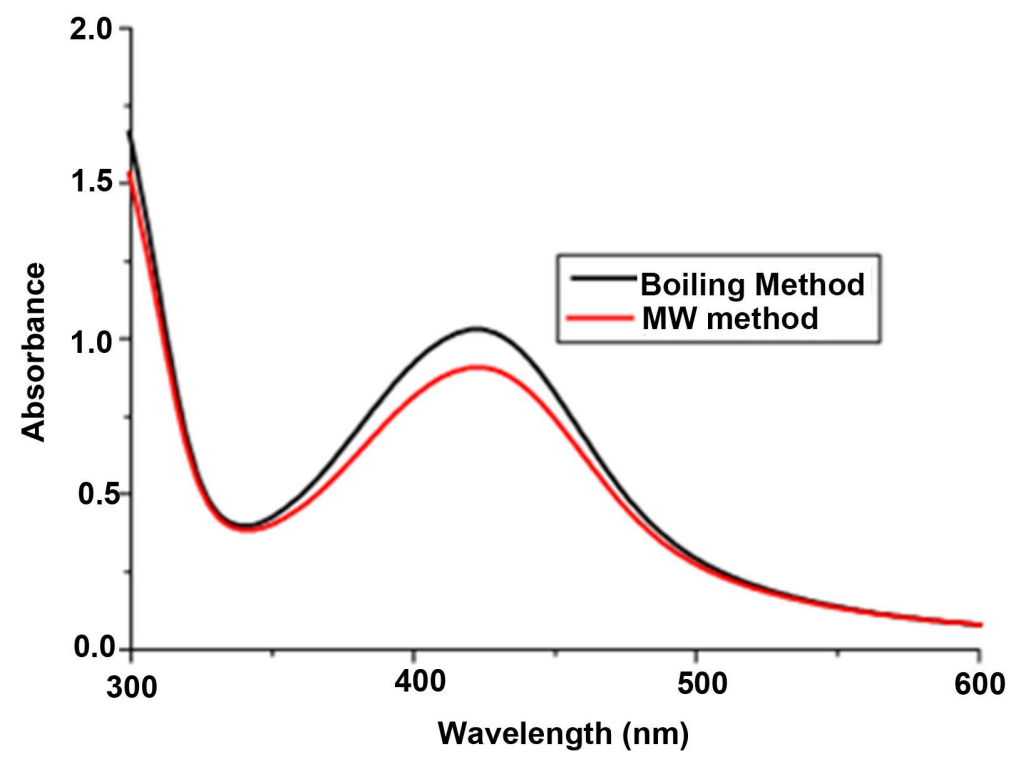

Figure 1. Absorption spectra of synthesized Ag NPs in water with different heating techniques. 

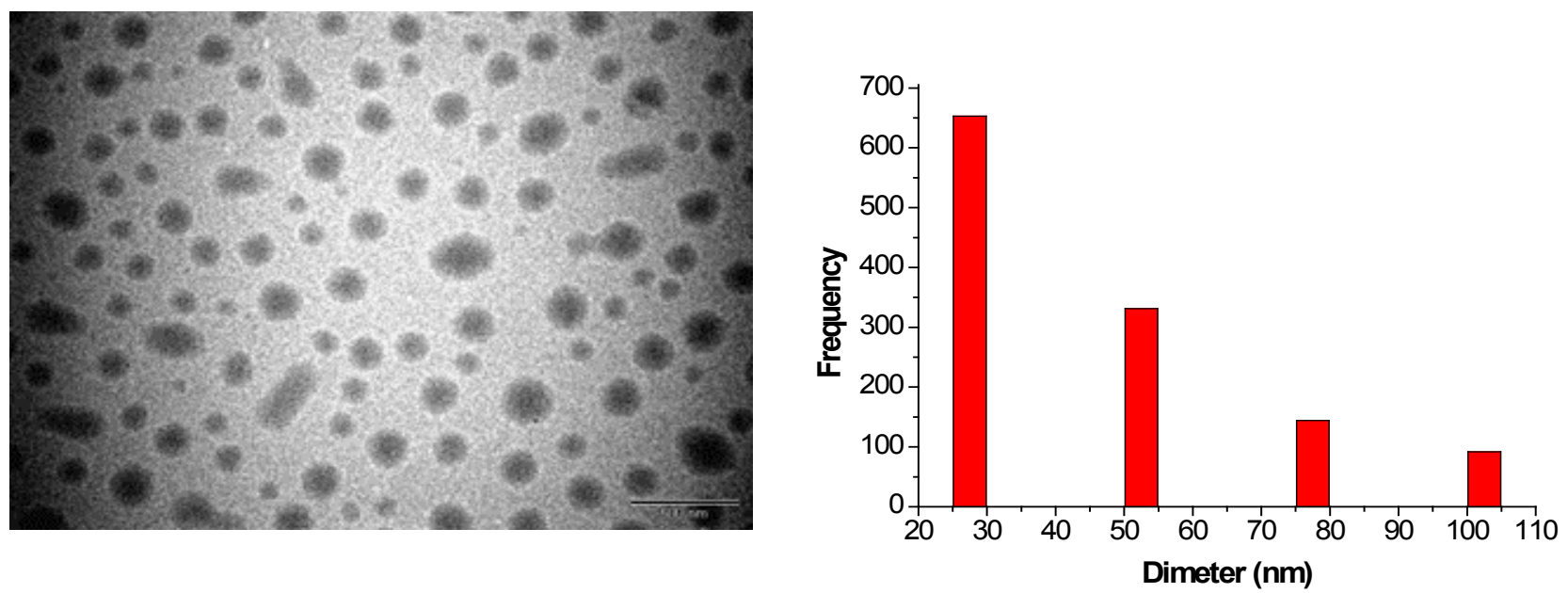

(a)
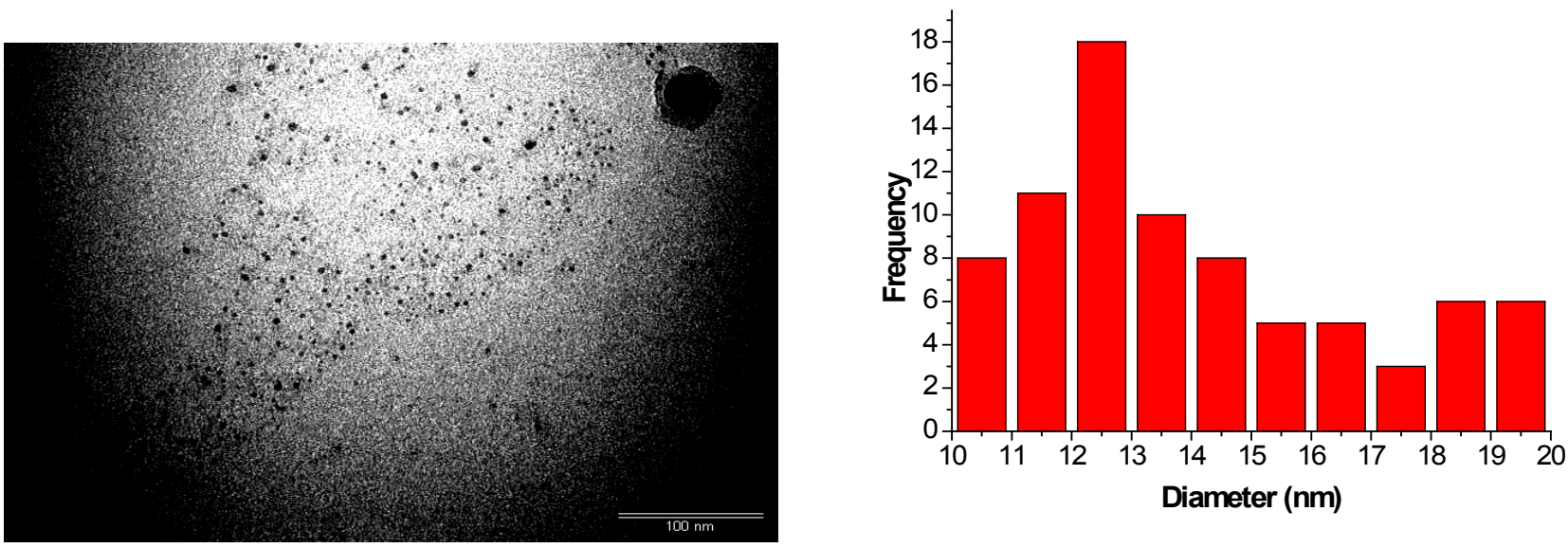

(b)

Figure 2. Transmission Electron Microscopy image and corresponding particle size distribution using J-image software (a) boiling method. (b) Microwave method.

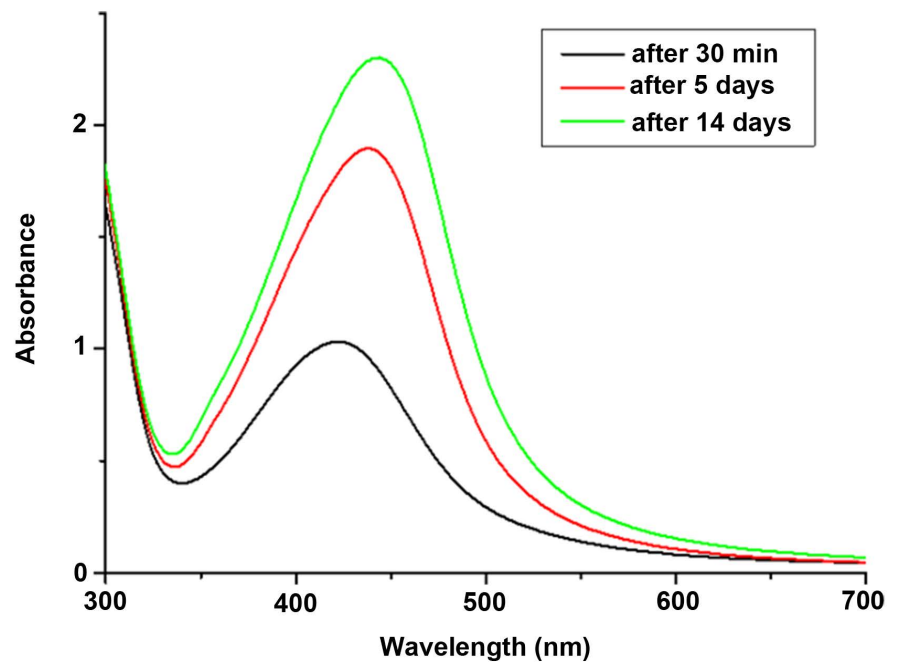

Figure 3. Variation of the SPR absorption peak with respect to the time for Ag nanoparticles prepared by boiling method. 
electrostatic repulsion force and a weak Van-der Waals attraction force [10]. Transmission Electron Microscopy (TEM) was performed for determining shapes and sizes of the synthesized Ag nanoparticles. The samples were prepared by dispersing a few drops of the solution on a copper grid and then at room temperature to dry. The TEM study reveals that the size and shape of most nanoparticles are nearly spherical. The size distribution of nanoparticles was analyzed using Image $1.5 \mathrm{~J}$ software. This was performed by converting pixels on the TEM images into nanometers by applying the scale of the image. Figure 3 shows TEM image and size distribution histogram of AgNPs. It can be seen that the nanoparticles have wide distribution range with a high yield of size, from 25 $\mathrm{nm}$ for nanoparticles synthesized by boiling method (a) to $15 \mathrm{~nm}$-size for nanoparticles synthesized by Microwave method (b).

\section{Results and Discussions}

\subsection{Z-Scan Measurements}

The Z-scan technique was used to investigate the nonlinear optical properties of green synthesized Ag nanoparticles. This technique relies on the fact that the intensity varies along the optical axis of the convex lens and it is maximum at the focus. The Z-scan experiment was performed using Argon ion laser $(488 \mathrm{~nm}$, $514 \mathrm{~nm}$, power $20 \mathrm{~mW}$ ) and $\mathrm{HeNe}$ laser $(632.8 \mathrm{~nm}$, power $20 \mathrm{~mW})$. A lens of 50 $\mathrm{mm}$ focal length was used to focus the laser beam, to roughly a beam waist of 20 $\mu \mathrm{m}$. The sample can be moved along the $\mathrm{z}$-axis by a computer-controlled motorized translational stage. The transmittance of the samples was measured with and without aperture (closed and open scan) in the far-field of the lens, as the sample moved through the focal point. The closed case gives the information about nonlinear refractive index while the open one gives information on nonlinear absorption coefficient.

Figure 4 shows the normalized transmittance for the open aperture case for the sample size of $15 \mathrm{~nm}$ at an excitation wavelength of $488 \mathrm{~nm}$. The transmittance is symmetrical with respect to the focus $(Z=0)$, where it reaches a minimum transmission. This is an indication that the sample exhibits reverse saturation absorption, RSA. Similar characteristics were observed with the samples studied at $514 \mathrm{~nm}$ and $633 \mathrm{~nm}$.

The origin of nonlinear absorption in nanoparticles can be attributed to different processes such as two-photon absorption, inter-band, intra-band transitions, excited state absorption and nonlinear scattering. In our $\mathrm{Z}$ scan experiment, the excitation of the wavelengths was $488 \mathrm{~nm}(2.54 \mathrm{eV}), 514 \mathrm{~nm}(2.41 \mathrm{eV})$ and $633 \mathrm{~nm}(1.96 \mathrm{eV})$. The energies of these photons are much less than the electronic transition gap between the d-bands and the conduction band $(4.1 \mathrm{eV})$ for Ag nanoparticles [11] [12]. Therefore, the only possible transition would be through two-photon absorption process; therefore, the observed RSA in our study is attributed to this process. A slight mismatch between conduction band gap of Ag nanoparticles and the total energy of the two photons will be compensated by phonon-assisted excitation [13]. 


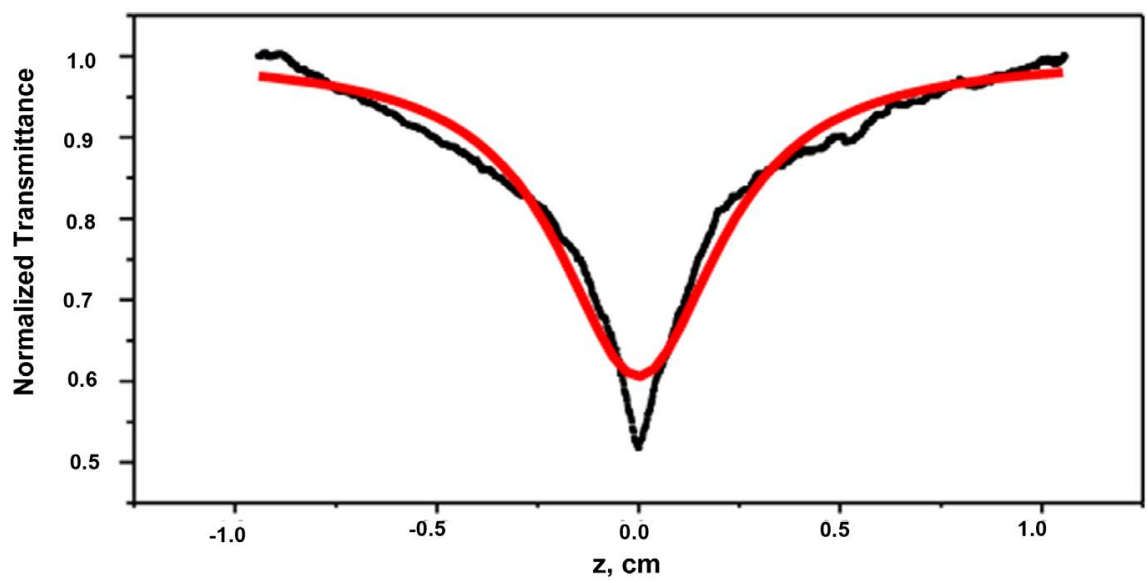

Figure 4. Open aperture Z-scan signal of Ag nanoparticles of diameter $15 \mathrm{~nm}$ at $488 \mathrm{~nm}$ and a line is a fit of equation 1 to experimental data.

For the open aperture case, the normalized transmittance is given by [14]:

$$
T=1-\frac{q_{0}}{1+x^{2}}
$$

where $x=z / z_{0}$ and $z_{0}=\pi \omega_{0}^{2} / \lambda$ is the diffraction length of the Gaussian beam, $\omega_{0}$ is the beam waist and $q_{0}$ is the nonlinear phase change that is related to the nonlinear absorption coefficient $\beta$ by:

$$
\beta=\frac{2 \sqrt{2} q_{0} \alpha}{I_{0}\left(1-\mathrm{e}^{-\alpha l}\right)}
$$

where $\alpha$ is the linear absorption coefficient ( $\alpha=$ Absorbance $\times \ln (10) / I$ ), $l$ is the thickness of the sample and $I_{0}$ is the peak laser irradiance at the focus $\left(I_{0}=2 P / \pi \omega_{0}^{2}\right)$ where $P$ is the laser power. A fit of Equation (1) to the experimental data is used to calculate the values of nonlinear absorption at different wavelengths for both samples. The results are shown in Table 1.

The nonlinear absorption coefficient $\beta$ is related to the imaginary part of the third order susceptibility through:

$$
\operatorname{Im}\left(\chi^{(3)}\right)=n^{2} \int_{0} c \lambda \beta / 2 \pi
$$

where $n$ is the index of refraction taken here to be $1.33, \epsilon_{0}$ is the free space permittivity. The calculated values of imaginary third order susceptibility are shown in Table 1.

In the case of closed aperture configuration, the normalized transmittance is given by [14]:

$$
T(z)=1+\frac{4 \Delta \varphi x}{\left(1+x^{2}\right)\left(9+x^{2}\right)}
$$

where $\Delta \varphi$ is the laser-induced phase shift that is related to the nonlinear refractive coefficient $n_{2}$ by the Equation (5).

$$
n_{2}=\frac{\Delta \varphi \lambda \alpha}{2 \pi I_{0}\left(1-\mathrm{e}^{-\alpha l}\right)}
$$


Table 1. Nonlinear absorption, nonlinear refractive index and nonlinear susceptibility values of Ag nanoparticles measured for different wavelengths.

\begin{tabular}{lcccccccc}
\hline Sample (size) & $\lambda, \mathrm{nm}$ & Power $(\mathrm{mW})$ & $\beta(\mathrm{cm} / \mathrm{W}) \times 10^{-3}$ & $\Delta \varphi(\mathrm{rad})$. & $\mathrm{n}_{2}\left(\mathrm{~cm}^{2} / \mathrm{W}\right) \times 10^{-7}$ & $\begin{array}{c}\operatorname{Im}\left(\chi^{(3)}\right) \times 10^{-14} \\
\left(\mathrm{~m}^{2} / \mathrm{V}^{2}\right)\end{array}$ & $\begin{array}{c}\mathrm{Re}\left(\chi^{(3)}\right) \times \\
10^{-14}\left(\mathrm{~m}^{2} / \mathrm{V}^{2}\right)\end{array}$ & $\begin{array}{c}\mathrm{I} \chi^{(3)} \times 10^{-14} \\
\left(\mathrm{~m}^{2} / \mathrm{V}^{2}\right)\end{array}$ \\
\hline 1) $\mathrm{Ag}(15 \mathrm{~nm})$ & 514.5 & 18 & 5.2 & - & - & 2.0 & - & - \\
2) $\mathrm{Ag}(25 \mathrm{~nm})$ & 514.5 & 18 & 10.7 & 1.02 & 0.73 & 4.1 & 6.9 & 8.0 \\
$7) \mathrm{Ag}(15 \mathrm{~nm})$ & 488 & 24 & 1.9 & 1.27 & 0.53 & 0.7 & 5.0 & 5.0 \\
6) $\mathrm{Ag}(25 \mathrm{~nm})$ & 488 & 24 & 4.1 & 3.26 & 2.34 & 1.5 & 22 & 22 \\
9) $\mathrm{Ag}(25 \mathrm{~nm})$ & 632.8 & 17 & 0.4 & 0.42 & 0.16 & 0.2 & 1.5 & 1.5 \\
\hline
\end{tabular}

The nonlinear refractive index was measured by the fitting of Equation (4) to the experimental data. The values of the nonlinear refractive index for all samples are shown in Table 1.

The refractive nonlinear coefficient is related to the real part of the third order susceptibility through:

$$
\operatorname{Re}\left(\chi^{(3)}\right)=2 n^{2} \int_{0} c n_{2}
$$

The absolute value of $\chi^{(3)}$ is then given by:

$$
|\chi|^{(3)}=\left(\operatorname{Im}\left(\chi^{(3)}\right)^{2}+\operatorname{Re}\left(\chi^{(3)}\right)^{2}\right)^{1 / 2}
$$

The calculated values of real and the absolute third order susceptibility are shown in Table 1.

Figure 5 shows a typically normalized transmittance at wavelengths $514 \mathrm{~nm}$ (closed Z-scan) for the $25 \mathrm{~nm}$-size NP's as a function of the sample's position. The normalized transmittance curves for our samples were characterized by a pre-focal peak followed by a post-focal valley. This peak-valley configuration implies that the nonlinear refractive index of the solution is negative $\left(n_{2}<0\right)$ (self-defocusing). Similar characteristics were shown by the sample studied at $488 \mathrm{~nm}$ and at $633 \mathrm{~nm}$. The observed asymmetric nature of the Z-scan measurements along with the fact that the laser beam used in the experiment is a $\mathrm{CW}$ mode, together with peak-valley configuration suggests that the nonlinear refractive index observed is of thermal-origin [15] [16]. The nonlinear refractive index for all samples, in this case, may be attributed to a thermal nonlinearity resulting from the formation of the thermal lens in the medium around metal nanoparticles due to the effective heat transfer from the nanoparticles to the medium.

The nonlinear optical properties of Ag nanoparticles samples prepared by boiling and Microwave methods were investigated. For both samples, reverse saturation was observed. The nonlinear absorption and the nonlinear refractive index of samples found to be higher for $25 \mathrm{~nm}$ than $15 \mathrm{~nm}$ sample by a factor 2 and 4 respectively at the studied excitation wavelengths. These differences may arise from the variation of the nanoparticle sizes to the nanoparticle sizes as explained above. In fact, the change in sizes leads to a slight change in the energy 


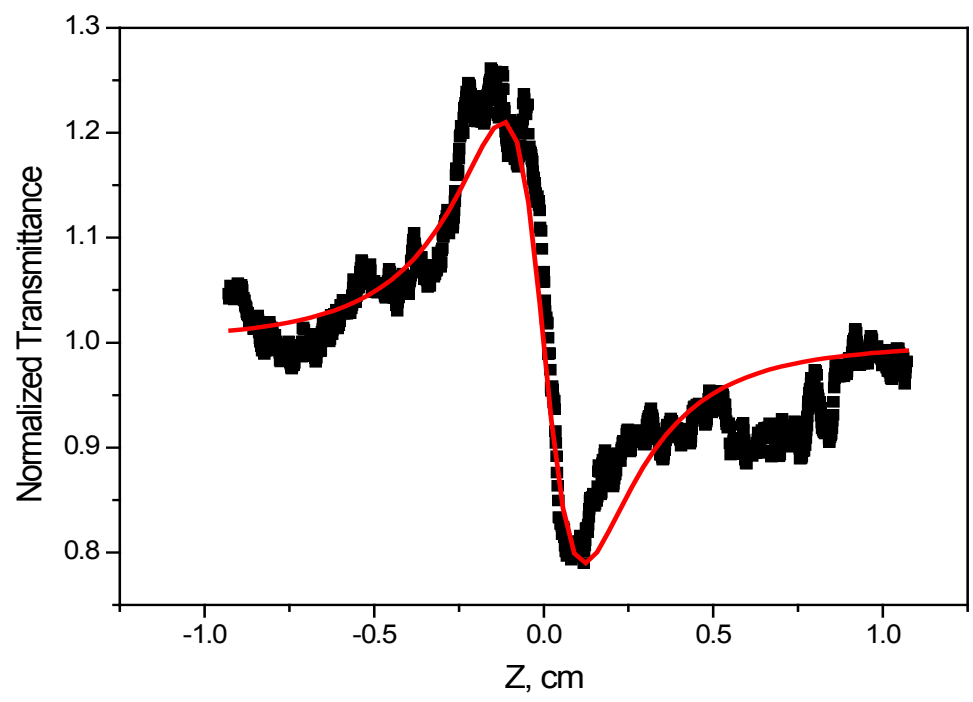

Figure 5. Closed aperture z-scan for Ag nanoparticles of size $25 \mathrm{~nm}$ at 514.5 $\mathrm{nm}$ and alineis a fit of equation 4 to experimental data.

band gap [10]. The wavelengths used in this experiment are close to SPR peak corresponding to $2.41 \mathrm{eV}$ to $1.96 \mathrm{eV}$ which is close to the resonance (one photon absorption) corresponding to SPR peak at $433 \mathrm{~nm}(2.87 \mathrm{eV})$, therefore one photon absorption cannot be ignored. As mentioned above, the nonlinearity observed, in this case, is due to the two-photon absorption which reveals that two-photon excitation cross section is enhanced due to resonant one-photon absorption. A mismatch between conduction band gap of Ag nanoparticles and one by photon energy will be compensated by phonon-assisted excitation [12]. The enhancement also arises due to an increased optical nonlinear interaction between the incident photon and the nanoparticles particles. This can be explained by the fact that, with an increase in particle size, there is an increase in the multiple scattering from the Ag nanoparticles which leads to a large effective interaction length whichin turn results in an enhancement in nonlinear absorption [12]. The nonlinear absorption and nonlinear refractive index values observed in this experiment are in the same order of magnitude with the reported values by Shahriari et al. [17] [18] [19] and one order of magnitude lower than those reported by Faraji et al. [20].

\subsection{Optical Switching}

A pump and probe experiment was used to demonstrate optical switching. A laser beam from an Argon ion laser $(\lambda=514 \mathrm{~nm}, 20 \mathrm{~mW})$ was used as a pump beam and a very low power laser beam from a He-Ne laser $(\lambda=633 \mathrm{~nm})$ was used as the probe beam. Both beams were overlapped at the focus of a lens. The probe beam was isolated from the pump beam using a narrow band filter. The choice of these two laser wavelengths is based on the fact that the ground-state absorption at $632.8 \mathrm{~nm}$ is weak (see absorption spectra above). The experiment was performed by moving the sample to a position beyond the valley as demon- 
strated by closed z-scan experiment. The pump beam was modulated with a mechanical chopper. The input of the pump beam and probe beam transmission was detected by a photodiode and displayed on the oscilloscope. Figure 6 shows the traces of the waveforms for the pump beam (black trace) and the probe beam (red trace). It is clear from the figure that when the pump beam is in "on state", the probe beam is intensively absorbed and is in "off state. This phenomenon is probably related to the presence of a strong nonlinearity observed at $514.5 \mathrm{~nm}$.

\subsection{Optical Limiting}

The observed nonlinear refractive index is used to demonstrate the power limiting performance. Silver nanoparticles in distilled water prepared boiling method are placed in $1 \mathrm{~mm}$ cuvette. In order to obtain the best performance, the sample is placed near post valley. The output power of the Argon Ion laser was recorded as the power of the incident beam is varied with neutral density filters. Figure 7 shows the optical limiting behavior of Ag nanoparticles at $514 \mathrm{~nm}$. It can be clearly seen from the graph that the transmitted output powers show no or a small variation at low incident power. At high incident powers, the output power shows a large variation compared to the transmitted output at low power irradiance. The optical limiting threshold was found to be $0.1 \mathrm{~mW}$. The low power limiting threshold observed here is may be due to a contribution of the thermal effect which may arise due to the nature of the laser used in this experiment. The optical limiting can be explained as follows: The localized absorption of the focused Gaussian beam by the sample produces a spatial distribution of the Temperature in the sample solution which results in spatial variation of refractive index [14] [15]. This light-induced change of refractive index can have a considerable effect on the laser propagation in the nonlinear medium, which leads to the well-known self-action effect phenomena such as self-defocusing, self-focusing.

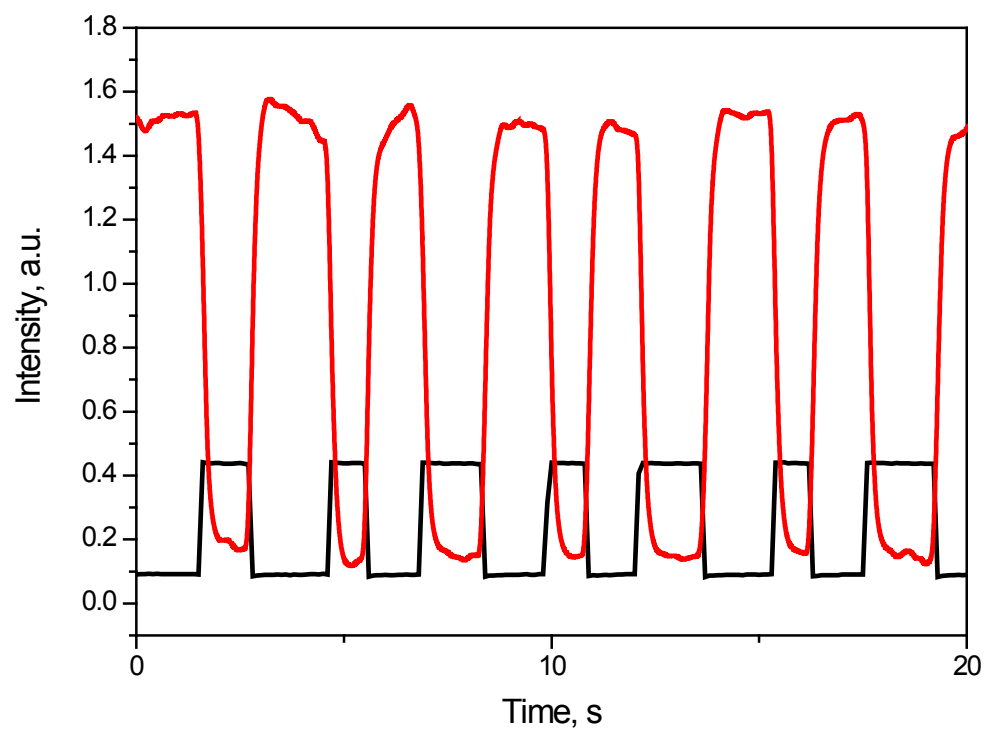

Figure 6. All-Optical switch inverter. 


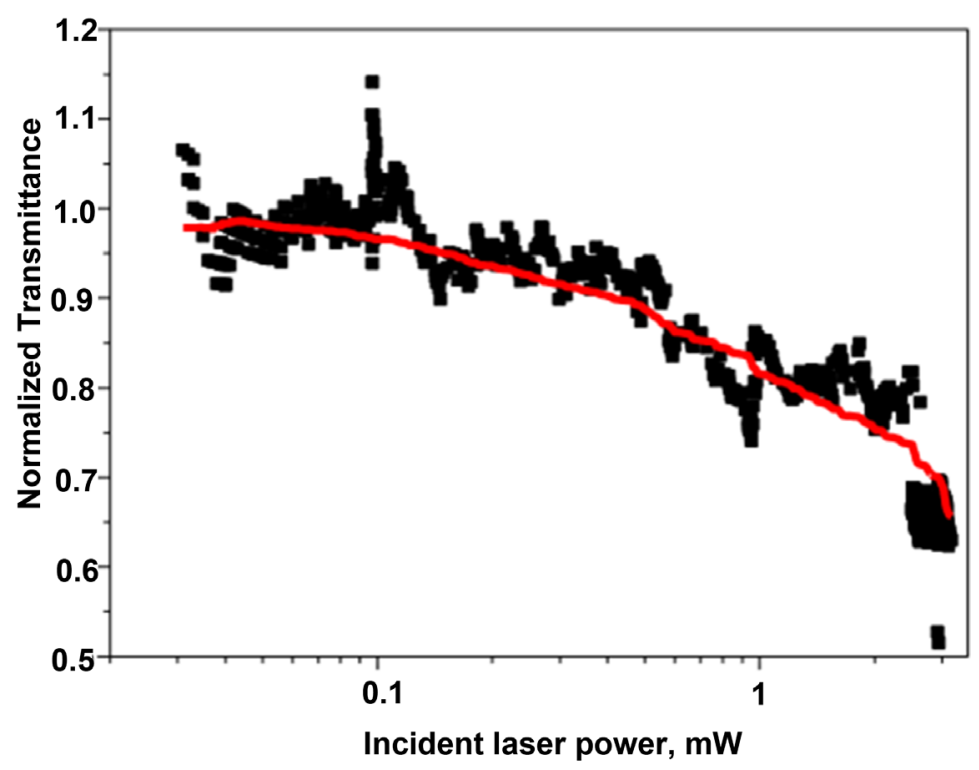

Figure 7. The optical limiting behaviour of Ag nanoparticle of size $25 \mathrm{~nm}$ at an excitation wavelength of $514.5 \mathrm{~nm}$.

Therefore, the observed nonlinear effect can be attributed to the thermal nonlinearity resulting from absorption of the laser beam used in the experiment by the AgNP sample at this wavelength. Further experiments with pulsed laser are needed to quantify the contribution of electronic versus thermal effects and are in progress.

\section{Conclusion}

Ag NP's were synthesized using aqueous extract cinnamon berks. Microwave heating and boiling methods were used for preparation. The stability of Ag NPS was monitored over a month using a UV-Vis spectrometer. The green synthesized nanoparticles showed nonlinear properties. These properties were used to demonstrate optical limiting and optical switching. The green synthesized Ag nanoparticles using cinnamon is quick, stable, and safe and can be used for optical devices and medical applications.

\section{Acknowledgements}

The authors would like to thank institution for Research and Medical Consultation, Imam Abdul-Rahman Bin Faisal University, Dammam, Saudi Arabia for TEM technical support. In addition, the authors would like to thank Dr. Hessah Al Muzafer, Chemistry Department, College of Sciences, Imam Abdul-Rahman Bin Faisal University, and Dammam, Saudi Arabia for her help. We thank full to the school of postgraduate studies and research for financial support.

\section{References}

[1] Amendola, V. and Meneghetti, M. (2009) Laser Ablation Synthesis in Solution and 
Size Manipulation of Noble Metal Nanoparticles. Physical Chemistry Chemical Physics. PCCP, 11, 3805-3821. https://doi.org/10.1039/b900654k

[2] Zhao, Y., Jiang, Y. and Fang, Y. (2006) Spectroscopy Property of Ag Nanoparticles. Spectrochimica Acta Part A, Molecular and Biomolecular Spectroscopy, 65, 1003-1006. https://doi.org/10.1016/j.saa.2006.01.010

[3] Wang, H., Qiao, X., Chen, J. and Ding, S. (2005) Preparation of Silver Nanoparticles by Chemical Reduction Method. Colloids and Surfaces A: Physicochemical and Engineering Aspects, 256, 111-115. https://doi.org/10.1016/j.colsurfa.2004.12.058

[4] Bindhu, M.R. and Umadevi, M. (2013) Synthesis of Monodispersed Silver Nanoparticles Using Hibiscus cannabinus Leaf Extract and Its Antimicrobial Activity. Spectrochimica Acta Part A: Molecular and Biomolecular Spectroscopy, 101, 184-190. https://doi.org/10.1016/j.saa.2012.09.031

[5] El Khoury, E., Abiad, M., Kassaify, Z.G. and Patra, D. (2015) Green Synthesis of Curcumin Conjugated Nanosilver for the Applications in Nucleic Acid Sensing and Anti-Bacterial Activity. Colloids and surfaces B, Biointerfaces, 127, 274-280. https://doi.org/10.1016/j.colsurfb.2015.01.050

[6] Kiruba Daniel, S.C.G., Mahalakshmi, N., Sandhiya, J., Nehru, K. and Sivakumar, M. (2013) Rapid Synthesis of Ag Nanoparticles Using Henna Extract for the Fabrication of Photoabsorption Enhanced Dye-Sensitized Solar Cell (PE-DSSC). Advanced Materials Research, 678, 349-360. https://doi.org/10.4028/www.scientific.net/AMR.678.349

[7] Zhang, Y.-X. and Wang, Y.-H. (2017) Nonlinear Optical Properties of Metal Nanoparticles: A Review. RSC Advances, 7, 45129-45144. https://doi.org/10.1039/C7RA07551K

[8] Abdalla, K.H., Al-Hannan, F., Alghamdi, A. and Henari, F.Z. (2017) Green Synthesis of Silver Nanoparticles Using Cinnamon (Cinnamomum cassia), Characterization and Antibacterial Activity. International Journal of Science and Research (IJSR), 6, 965-971.

[9] Mogensen, K.B. and Kneipp, K. (2014) Size-Dependent Shifts of Plasmon Resonance in Silver Nanoparticle Films Using Controlled Dissolution: Monitoring the Onset of Surface Screening Effects. The Journal of Physical Chemistry C, 118, 28075. https://doi.org/10.1021/jp505632n

[10] Prathna, T.C., Chandrasekaran, N., Raichur, A.M. and Mukherjee, A. (2011) Biomimetic Synthesis of Silver Nanoparticles by Citrus limon (Lemon) Aqueous Extract and Theoretical Prediction of Particle Size. Colloids and Surfaces B, Biointerfaces, 82, 152-159. https://doi.org/10.1016/j.colsurfb.2010.08.036

[11] Gurudas, U., Brooks, E., Bubb, D.M., Heiroth, S., Lippert, T. and Wokaun, A. (2008) Saturable and Reverse Saturable Absorption in Silver Nanodots at $532 \mathrm{~nm}$ Using Picosecond Laser Pulses. Journal of Applied Physics, 104, Article ID: 073107. https://doi.org/10.1063/1.2990056

[12] Henari, F.Z. and Dakhel, A.A. (2011) Observation of Simultaneous Reverse Saturation Absorption and Saturation Absorption in Silver Nanoparticles Incorporated into Europium Oxide Thin Film. Optics Communications, 284, 651-655. https://doi.org/10.1016/j.optcom.2010.09.090

[13] Qian, W., Yan, H., Wang, J.J. and Zou, Y.H. (1999) Observation of Coherent Phonons in Silver Nanoparticles Embedded in $\mathrm{BaO}$ Thin Films. Applied Physics Letters, 74, 1806. https://doi.org/10.1063/1.123092

[14] Sheik-Bahae, M., Said, A.A., Wei, T.-H., Hagen, D.J. and Van Stryland, E.W. (1990) Sensitive Measurement of Optical Nonlinearities Using a Single Beam. IEEE Journal 
of Quantum Electronics, 26, 760-769.

[15] Jacinto, C., Messias, D.N., Andrade, A.A., Lima, S.M., Baesso, M.L. and Catunda, T. (2006) Thermal Lens and Z-Scan Measurements: Thermal and Optical Properties of Laser Glasses-A Review. Journal of Non-Crystalline Solids, 352, 3582-3597. https://doi.org/10.1016/j.jnoncrysol.2006.04.025

[16] Gómez, S.L., Cuppo, F.L.S., Neto, A.M.F., Kosa, T., Muramatsu, M. and Horowicz, R.J. (1999) Z-Scan Measurement of the Nonlinear Refractive Indices of Micellar Lyotropic Liquid Crystals with and without the Ferrofluid Doping. Physical Review $E, 59,3059$.

[17] Shahriari, E., Yunus, W.M. and Zamiri, R. (2013) The Effect of Nanoparticle Size on Thermal Diffusivity of Gold Nano-Fluid Measured Using Thermal Lens Technique. Journal of the European Optical Society_Rapid Publications, 8, Article ID: 13026.

[18] Shahriari, E., Yunus, W.M. and Naghavi, K. (2010) Z-Scan Determination of Two-Photon Absorption in Ag Nanofluid Prepared by $\gamma$-Radiation Methods. Journal of Optoelectronics and Advanced Materials, 12, 1676-1679.

[19] Majles Ara, M.H., Javadi, Z. and Sirohi, R.S. (2011) Measurement of Nonlinear Refractive Index of $\mathrm{Ag}$ and $\mathrm{Au}$ Nanoparticles Using Moire Technique. $O p$ tik-International Journal for Light and Electron Optics, 122, 1961-1964.

https://doi.org/10.1016/j.ijleo.2010.11.025

[20] Faraji, N., Mat Yunus, W.M., Kharazm, A., Saion, E., Shahmiri, M. and Tamchek, N. (2012) Synthesis, Characterization and Nonlinear Optical Properties of Silver/PVA Nanocomposites. Journal of the European Optical Society-Rapid Publication, 7, Article ID: 12040. https://doi.org/10.2971/jeos.2012.12040 